\title{
Dissipation in Relativistic Pair-plasma Reconnection: Revisited
}

\author{
Seiji Zenitani \\ Research Institute for Sustainable Humanosphere, Kyoto University, Gokasho, \\ Uji, Kyoto 611-0011, Japan \\ E-mail: zenitani@rish.kyoto-u.ac.jp
}

\begin{abstract}
Basic properties of relativistic magnetic reconnection in electronpositron pair plasmas are investigated by using a particle-in-cell (PIC) simulation. We first revisit a problem by Hesse \& Zenitani (2007), who examined the kinetic Ohm's law across the $\mathrm{X}$ line. We formulate a relativistic Ohm's law by decomposing the stress-energy tensor. Then, the role of the new term, called the heat-flow inertial term, is examined in the PIC simulation data. We further evaluate the energy balance in the reconnection system. These analyses demonstrate physically transparent ways to diagnose relativistic kinetic data.
\end{abstract}

\section{Introduction}

Magnetic reconnection is a fundamental plasma process to drive explosive events in space and astrophysical environments [1]. To allow the change in the field-line topology, the ideal condition for arbitrary plasma species $s$,

$$
\vec{E}+\vec{V}_{s} \times \vec{B}=0,
$$

should be violated around the $\mathrm{X}$ line, across which the magnetic flux is transported. In a collisionless plasma, the central problems in reconnection physics are how the ideal condition is violated and what kinetic mechanisms are responsible for the violation. This issue is often referred to as the dissipation problem, because the violation of the ideal condition is necessary for the nonideal energy dissipation that drives magnetic reconnection.

In a nonrelativistic plasma, significant efforts have been paid to the electron idealness, because the electrons are the last species to decouple from the field lines near the $\mathrm{X}$ line. The electron Ohm's law, obtained from the electron momentum equation, is useful to analyze the problem,

$$
\vec{E}+\vec{V}_{e} \times \vec{B}=-\frac{1}{e n_{e}} \nabla \cdot \mathbb{P}_{e}-\frac{m_{e}}{e}\left(\frac{\partial \vec{V}_{e}}{\partial t}+\left(\vec{V}_{e} \cdot \nabla\right) \vec{V}_{e}\right),
$$

where $\mathbb{P}_{e}$ is the electron pressure tensor. In two-dimensional quasisteady systems, only the first terms in the left and right hand sides survive at the $\mathrm{X}$ line, and therefore the reconnection electric field is balanced by the divergence of the electron pressure tensor term [1. Cai \& Lee 2] showed that the pressure-tensor term balances the reconnection electric field on a sub-ion timescale in PIC simulations. Hesse et al. [3. have demonstrated with PIC simulations that the divergence of the off-diagonal 
parts of the electron pressure tensor sustains the reconnection electric field in a quasisteady stage. He and his colleagues have long studied electron kinetic physics that is responsible for the off-diagonal parts of the electron pressure tensor. Insights from their works were reviewed by Ref. [4].

Magnetic reconnection is also believed to occur in a relativistic plasma in highenergy astrophysical settings [5]. The question then arises: how the reconnection proceeds in a relativistic kinetic plasma? However, since relativistic plasma physics is far more complicated than nonrelativistic one, even an appropriate form of Ohm's law was not clear. Theorists have constructed several relativistic forms of Ohm's law [6, 7, 8, from fluid equations or multi-species Boltzmann equations.

Over the last decades, kinetic modeling of relativistic magnetic reconnection have been in progress $[9,10,11,12,13,14,15,16,17,18,19,20$. Although many of these studies focused on the stability of a current-sheet, particle acceleration, and radiative signatures, Hesse \& Zenitani [13] made the first attempt to examine the kinetic Ohm's law near the X line in PIC simulations. Employing Wright \& Hadley 21]'s definition of the pressure tensor, they formulated a relativistic Ohm's law from the Vlasov equation. Then, they analyzed PIC simulation data of magnetic reconnection in a relativistic pair plasma, and found that the divergence of the "pressure tensor" term sustains the reconnection electric field at the $\mathrm{X}$ line, as in nonrelativistic cases. This or equivalent analysis has been employed by subsequent studies [14, 15, 18, 19. However, there were some problems in the framework in Ref. [13. First, the Wright-Hadley pressure tensor [21] was not symmetric. An asymmetric pressure tensor is not the one that we were looking for. Second, physical meanings of the terms in Ohm's law were not adequately given.

Another important issue in reconnection physics is the energy balance around the reconnection site. Although this is a very fundamental problem, scientists have started to discuss the energy balance very recently. Using MHD simulations, Birn et al. 22, 23, have argued that the reconnection process converts the incoming magnetic energy flux into the enthalpy flux in the outflow region. Successive hybrid and PIC simulations 24, 25, 26, 27, reported that the energy is mostly transferred to the enthalpy flux in the kinetic regimes as well. In a relativistic plasma, using relativistic two-fluid simulations, Zenitani et al. 28] demonstrated that the enthalpy flux carries most of the outgoing energy in antiparallel reconnection and that Poynting flux replaces the enthalpy flux in the presence of the out-of-plane guide field. To the best of our knowledge, no one has studied the energy balance in relativistic reconnection in depth with first-principle PIC simulations, because researchers focused on other important issues and because the analysis framework was not well known.

The purpose of this paper is to re-examine the Ohm's law problem in Ref. [13, based on our up-to-date knowledge. Decomposing the stress-energy tensor, we derive a relativistic kinetic form of Ohm's law, which contains a "heat-flow inertial term." We further apply the decomposition to the energy balance problem around the reconnection site.

This paper is organized as follows. In Section 2, we describe basic issues of relativistic mechanics and then we derive a relativistic extension of the electron Ohm's law. In Section 3, we describe the setup of our PIC simulation. In Section 4, we present the simulation results. The relativistic Ohm's law is evaluated. In Section 5 , we further extend our discussion to the energy balance problem during magnetic reconnection. Section 6 contains discussions and a summary. 


\section{Preparation}

Let us briefly review basic issues of relativistic statistical mechanics. The metric tensor is set to $g^{\alpha \beta}=\operatorname{diag}(-1,1,1,1)$. We set $c=1$ throughout the paper, but we sometimes keep $c$ to emphasize its physical meaning. The particle flux density $N^{\alpha}$ and the stress-energy tensor $T^{\alpha \beta}$ are defined in the following way,

$$
N^{\alpha}=\int f(u) u^{\alpha} \frac{d^{3} u}{\gamma}, \quad T^{\alpha \beta}=\int f(u) u^{\alpha} u^{\beta} \frac{d^{3} u}{\gamma} .
$$

We express the properties in the other frame by the prime sign $\left({ }^{\prime}\right)$. Since $d^{3} u / \gamma=$ $d^{3} u^{\prime} / \gamma^{\prime}$, both follow the Lorentz transformation,

$$
N^{\alpha}=\Lambda_{\mu}^{\alpha} N^{\prime \mu}, \quad T^{\alpha \beta}=\Lambda_{\mu}^{\alpha} \Lambda_{\nu}^{\beta} T^{\prime \mu \nu}
$$

where $\Lambda$ is the Lorentz transformation tensor.

The second-rank tensor $T$ can be decomposed in the following way [29]:

$$
T^{\alpha \beta}=\mathcal{E} u^{\alpha} u^{\beta}+q^{\alpha} u^{\beta}+q^{\beta} u^{\alpha}+P^{\alpha \beta}
$$

where $\mathcal{E} \equiv T^{\alpha \beta} u_{\alpha} u_{\beta}, q^{\alpha} \equiv-\Delta_{\beta}^{\alpha} T^{\beta \gamma} u_{\gamma}, P^{\alpha \beta} \equiv \Delta_{\gamma}^{\alpha} \Delta_{\delta}^{\beta} T^{\gamma \delta}$, and $\Delta^{\alpha \beta}=g^{\alpha \beta}+u^{\alpha} u^{\beta}$ is the projection operator. Here, $u^{\alpha}$ is an arbitrary flow, $\mathcal{E}$ is the energy density in the $u^{\alpha}$-moving frame, $q^{\alpha}$ and $P^{\alpha \beta}$ are projections of the energy flux and the pressure tensor in the flow frame. The four-vector $q^{\alpha}$ is called the heat flow. We expect $P^{\alpha \beta}=p u^{\alpha} u^{\beta}+p g^{\alpha \beta}$ for an ideal gas, where $p$ is a scalar pressure.

This decomposition works for an arbitrary four-velocity $u^{\alpha}$. For practical use, we need to choose an appropriate rest frame for the plasma flow. However, unlike in the nonrelativistic case, it is not straightforward to define the rest frame in a relativistic plasma. This is schematically illustrated in Figure 1. We assume that three particles travel in the $\pm x$ directions at relativistic speeds. Their speeds are $\sim c$, but they have different Lorentz factors. In this case, an average plasma number flow should be in

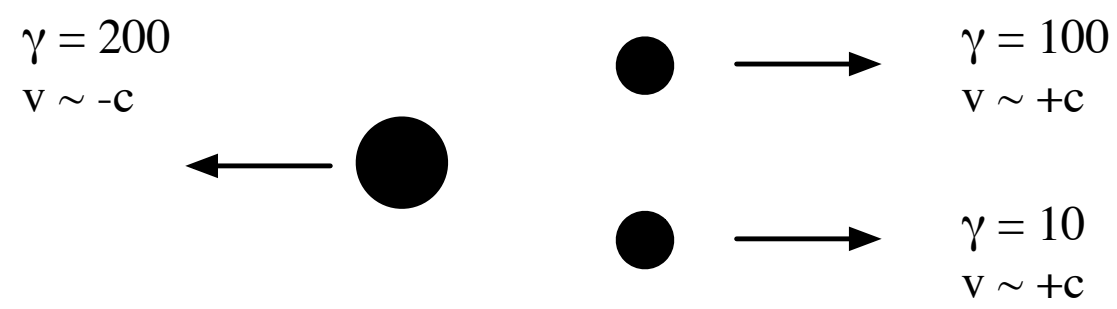

Figure 1. An example of a relativistic fluid system, which consists of three particles.

the right direction, while the energy obviously flows in the left direction. This tells us that there could be two rest frames, in which the plasma number flux is zero, and in which the plasma energy flux is zero. The former is called the Eckart frame [29], and the latter is called the Landau frame 30.

The Eckart-frame four-velocity $u_{(E)}^{\mu}$ can be straightforwardly obtained from the plasma number flux,

$$
u_{(E)}^{\mu}=N^{\mu} / \sqrt{-N^{\nu} N_{\nu}}=N^{\mu} / n,
$$

where $n=\sqrt{-N^{\nu} N_{\nu}}$ is the proper density. The Landau-frame four-velocity $u_{(L)}^{\mu}$ can be obtained from an eigenvalue problem of the stress-energy tensor [31, 20]. 
Our derivation will be presented in Appendix A. The Eckart and Landau frames are identical in the nonrelativistic regime, but it is important to distinguish the two frames in a relativistic plasma. In this paper, we employ the Eckart frame as the fluid rest frame. We assume $u^{\mu}=u_{(E)}^{\mu}$ unless stated otherwise.

We consider the energy momentum equation of the electron fluid,

$$
\partial_{\beta} T_{(e)}^{\alpha \beta}=-e F^{\alpha \beta} N_{\beta}=-e n F^{\alpha \beta} u_{\beta}
$$

where $F^{\alpha \beta}$ is the electromagnetic tensor. From the momentum $(i \beta)$ part of the equation, we obtain a relativistic generalization of the electron Ohm's law.

$$
\begin{gathered}
\vec{E}=-\vec{V} \times \vec{B}-\frac{1}{\gamma e n}\left(\partial_{t} T^{i 0}+\nabla \cdot\left(\mathcal{E} u^{i} u^{j}+Q^{i j}+P^{i j}\right)\right) \\
=-\vec{V} \times \vec{B}-\frac{1}{\gamma e n}\left(\partial_{t}\left(\gamma \mathcal{E} u^{i}+Q^{i 0}+P^{i 0}\right)\right. \\
\left.+\nabla \cdot\left(\mathcal{E} u^{i} u^{j}+Q^{i j}+P^{i j}\right)\right) .
\end{gathered}
$$

Here, $\vec{V}$ is the 3 -velocity for the electron Eckart velocity and $Q^{\alpha \beta} \equiv q^{\alpha} u^{\beta}+q^{\beta} u^{\alpha}$ is the heat-flow part of the stress-energy tensor (Eq. 5). In the detailed form (Eq. 9), the two $\mathcal{E}$-related terms of the right hand side correspond to the bulk inertial effect. They contain the effect of the relativistic gas temperature, because the energy is equivalent to the mass. For example, the term can be further split into the conventional part and an additional part by the relativistically hot gas, i.e., $\mathcal{E} u^{\alpha} u^{\beta}=n u^{\alpha} u^{\beta}+(\mathcal{E}-n) u^{\alpha} u^{\beta}$. The relativistic effects are included in (1) the temperature part of the bulk inertial term, (2) the heat-flow terms, (3) the time derivative of these terms, and (4) the Lorentz factors.

\section{Numerical model}

We carry out a two-dimensional particle-in-cell (PIC) simulation of relativistic magnetic reconnection. We consider a pair plasma of electrons and positrons. The Harris-Hoh current sheet is employed as an initial model [32, 33],

$$
\begin{aligned}
& B=B_{0} \tanh \left(\left[z-z_{0}\right] / L\right) \hat{x}, \\
& f_{s}(\vec{u})=\frac{n_{0} \cosh ^{-2}\left(\left[z-z_{0}\right] / L\right)}{4 \pi m^{2} c T K_{2}\left(m c^{2} / T\right)} \exp \left[\frac{-\gamma_{s}\left(\varepsilon-\beta_{s} m c u_{y}\right)}{T}\right] \\
& +\frac{n_{b g}}{4 \pi m^{2} c T K_{2}\left(m c^{2} / T\right)} \exp \left[-\frac{\varepsilon}{T}\right],
\end{aligned}
$$

where the subscript $s$ denotes the species ( $p$ for positrons and $e$ for electrons), the subscripts 0 and $b g$ denote physical quantities for the Harris-sheet plasmas and the uniform background plasmas, $L$ is the thickness of the current sheet, $K_{2}$ is the modified Bessel function of the second kind, $T$ is the proper temperature, $n_{0}$ is the proper density, $\varepsilon=\gamma m c^{2}$ is the particle energy, and $\beta_{s}$ is the drift speed of the Harris-sheet plasmas. We set $m=1, \beta_{p, e}= \pm 0.3$, and $T=m c^{2}$. The background density is set to $n_{b g}=0.2\left(\gamma_{e} n_{0}\right)$. Using the cell size $\Delta$, the Debye length and the current sheet thickness is set to $3 \Delta$ and $L=10 \Delta$. The time is normalized by the light crossing time of $L / c$. The plasma beta in the inflow region is set to 0.2 .

The system size is $1000 \Delta$ in $x$ and $600 \Delta$ in $z$. We consider two current sheets in the periodic system, as in our previous study [11]. The first Harris sheet is set at 
$z_{0}=450$ in the top half $(300<z<600)$. Then, the second Harris sheet is set at $z_{0}=150$ in the bottom half $(0<z<300)$. The magnetic polarities and the electric current are oppositely set for the second Harris sheet. In the paper, we present the results in the top half. Particles are initialized by a modified Sobol algorithm [34]. Magnetic reconnection is triggered by a small perturbation in a magnetic flux function. The maximum amplitude of the perturbed magnetic field is $\lesssim 0.1 B_{0}$.

To better evaluate the stress-energy tensor $T^{\alpha \beta}$, we employ several workarounds. First, we use a second-order shape factor in our PIC simulation. Second, we use $2 \times 10^{4}$ pairs of particles per cell for the Harris-sheet density $\gamma_{e} n_{0}$. Third, several properties are box-car averaged with neighboring cells.

\section{(a) Outflow speed ( $\mathrm{Vex}_{\text {ex }}$}

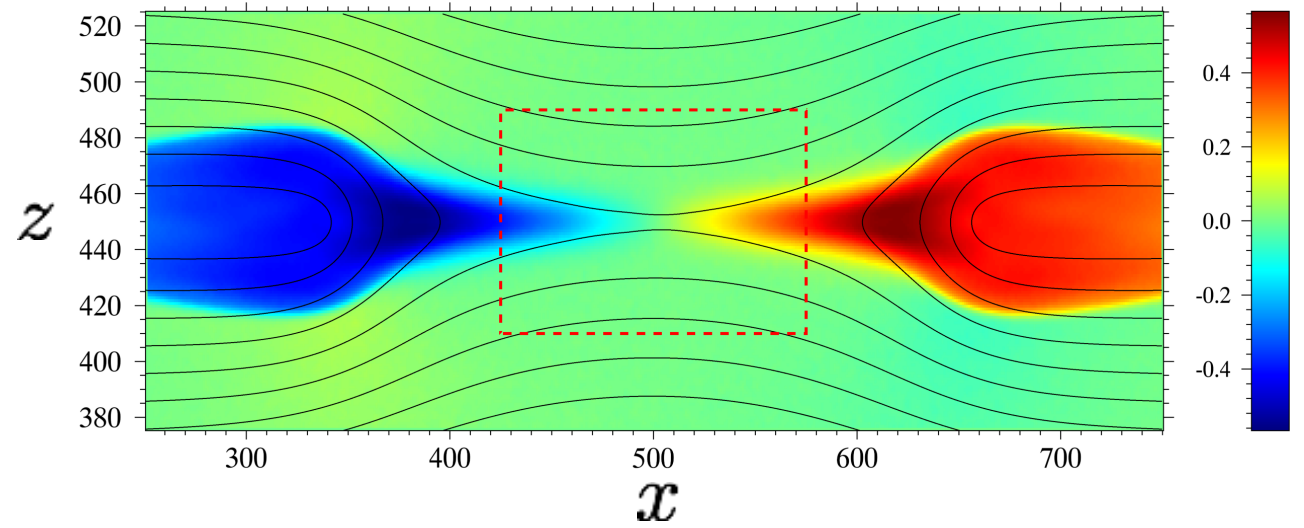

(b) Specific energy dissipation $\left(\mathrm{D}_{\mathrm{e}} / \mathrm{n}_{\mathrm{e}}\right)$

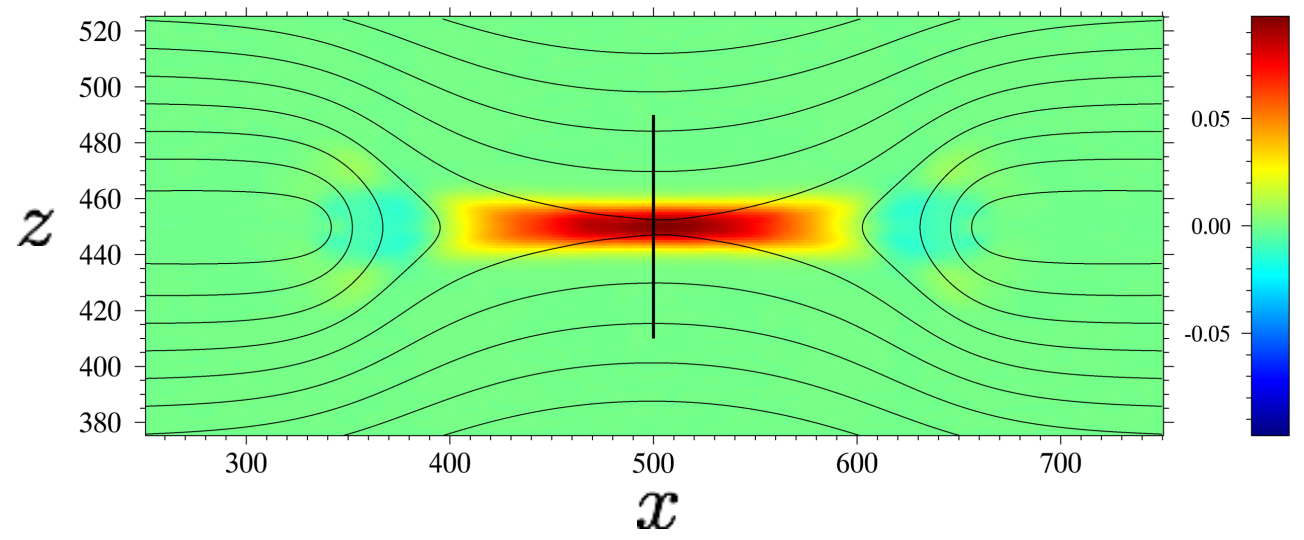

Figure 2. PIC simulation results at $t=85$. (a) Electron outflow $V_{e x}$. (b) Specific energy dissipation, $\mathcal{D}_{e} / n_{e}$ (Eq. 12 .

\section{Results}

The panels in Figure 2 present key properties at $t=85$. Figure2(a) shows the outflow component of the electron flow, $V_{e x}$. The bidirectional jets originate from the $\mathrm{X}$ line at the center. The electron maximum speed is $\pm 0.57 \mathrm{c}$. There are remnants of the 
initial current sheet, at $x \lesssim 350$ and $x \gtrsim 650$. The bottom panel shows a specific energy dissipation $\left(\mathcal{D}_{e} / n_{e}\right)$,

$$
\frac{1}{e c B_{0}}\left(\frac{\mathcal{D}_{e}}{n_{e}}\right) \equiv \frac{\gamma_{e}\left[\vec{J} \cdot\left(\vec{E}+\vec{V}_{e} \times \vec{B}\right)-\rho_{c} \vec{V}_{e} \cdot \vec{E}\right]}{e n_{e} c B_{0}},
$$

where $\rho_{c}$ is the electric charge-density. The $\mathcal{D}_{e}$ measure is proven to mark dissipation sites [35. In this study, we further divide it by the electron proper density $n_{e}$, in order to emphasize the dissipation region around the $\mathrm{X}$ line, while keeping $\mathcal{D}_{e} / n_{e}$ invariant. Thanks to the normalization constant $\left(e c B_{0}\right)^{-1}$, Equation 12 conveniently approximates the reconnection rate, $\mathcal{D}_{e} /\left(e n_{e} c B_{0}\right) \sim J_{y} E_{y} /\left(e n_{e} c B_{0}\right) \sim\left(E_{y} / B_{0}\right)$. The maximum value $\approx 0.1$ around the $\mathrm{X}$ line is comparable with the reconnection rate of this run.

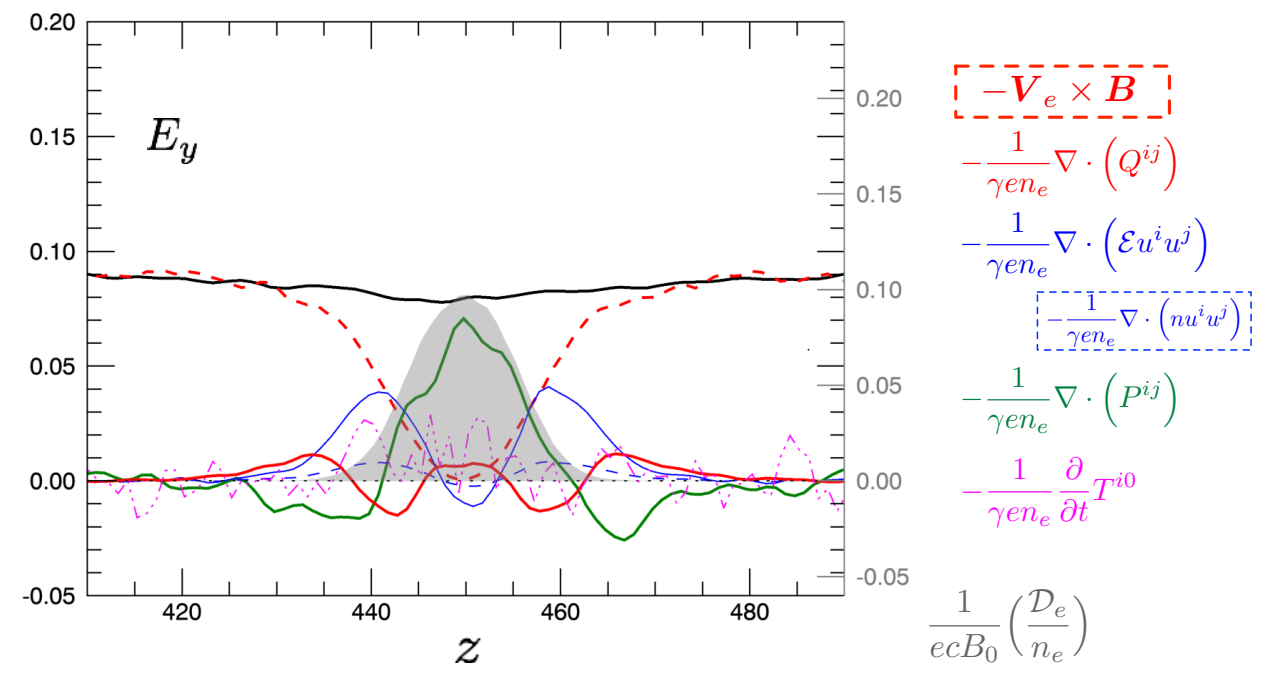

Figure 3. Composition of the reconnection electric field $E_{y}$ along the inflow line. The terms are based on Equation 8 The gray shadow shows the specific energy dissipation $\mathcal{D}_{e} / n_{e}$ (Eq. 12 , scaled by the right axis.

Using the PIC simulation data, we examine the violation of the electron idealness along the $x=500$ line, as indicated by the black line in Figure 2(b). Figure 3 shows the composition of the reconnection electric field $E_{y}$, based on Equation 8 . The time derivative parts were combined in $\partial_{t} T^{i 0}$, because we are more interested in the divergence part, which will persist in the quasisteady stage. In Figure 3 , the reconnection electric field (the black line) is in good agreement with the convection electric field (the red dashed line) in the inflow regions. As we approach the center, the bulk inertial term (blue) increases, which is partially canceled by the divergence of the pressure tensor (green). The blue dashed line shows the proper-mass part of the bulk inertial term, $\nabla \cdot\left(n_{e} u^{i} u^{j}\right)$. This is much smaller than the entire bulk inertial term. In this case, the plasma temperature increases from $T \approx 1$ in the upstream region to $\frac{1}{3} \operatorname{Tr}\left(P^{i j}\right) / n \gtrsim 1.5$ near the center, because plasmas are strongly energized around the reconnection site. Consequently, the relativistic pressure substantially enhances 
the bulk inertial effect. Near the center, the bulk inertial term becomes unimportant and then the pressure tensor term (green) is responsible for the reconnection electric field $E_{y}$. It is positive $-\frac{1}{\gamma e n_{e}} \nabla \cdot P^{i j}>0$ around $440 \lesssim z \lesssim 460$. This corresponds to the local momentum transport from the midplane $z \approx 450$. The central region corresponds to the site of the enhanced energy dissipation. The specific energy dissipation (Eq. 12 is indicated by the gray shadow in Figure 3. This suggests that the pressure-tensor term is the major contributor to the energy dissipation $\mathcal{D}_{e} \approx \gamma_{e} J_{y}\left(\vec{E}+\vec{V}_{e} \times \vec{B}\right)_{y} \approx-\left(J_{y} / e n_{e}\right)\left[\nabla \cdot\left(P^{i j}\right)\right]_{y}$ near the X line. In addition, we find some contributions from the temporal part of the inertial terms (magenta) and the divergence of the heat-flow tensor term (red). In particular, the heat-flow term only appears in the relativistic plasma. Hereafter we call it the heat-flow inertial term. Even though it is smaller than the pressure-tensor term, we noticed that it persists throughout the simulation run. It stems from the divergence of the off-diagonal parts of the electron heat-flow tensor, in particular from $-\partial_{z} Q_{e y z}$. Later in this section, we will further discuss the origin of the heat-flow inertial effect.

\section{(a) Pressure term (Peyz)}

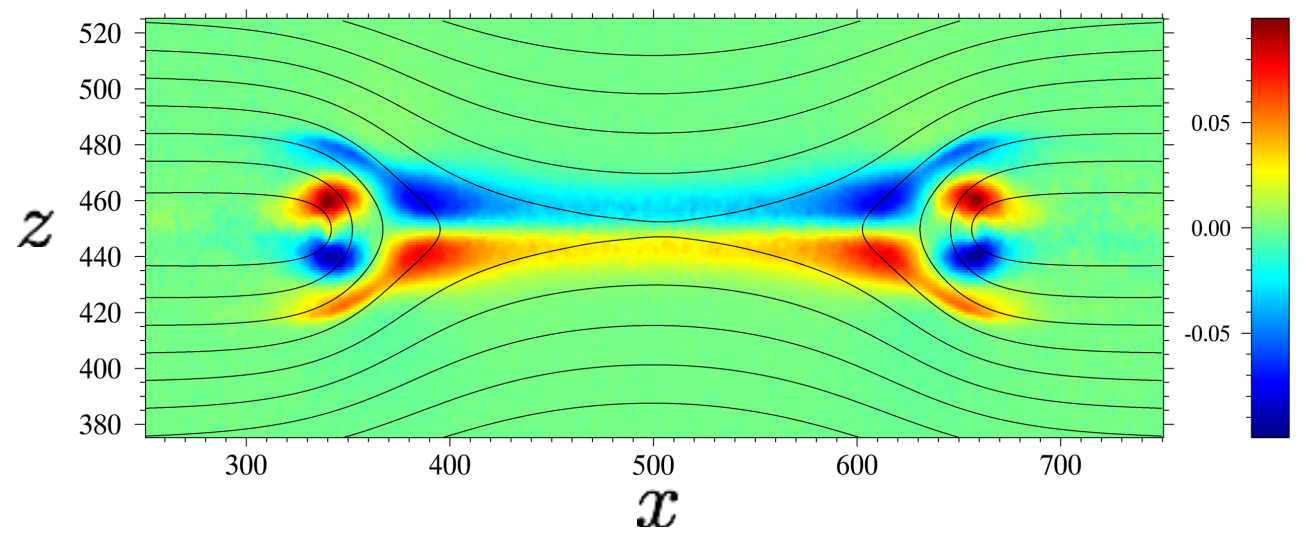

(b) Heat-flow term (Qeyz)

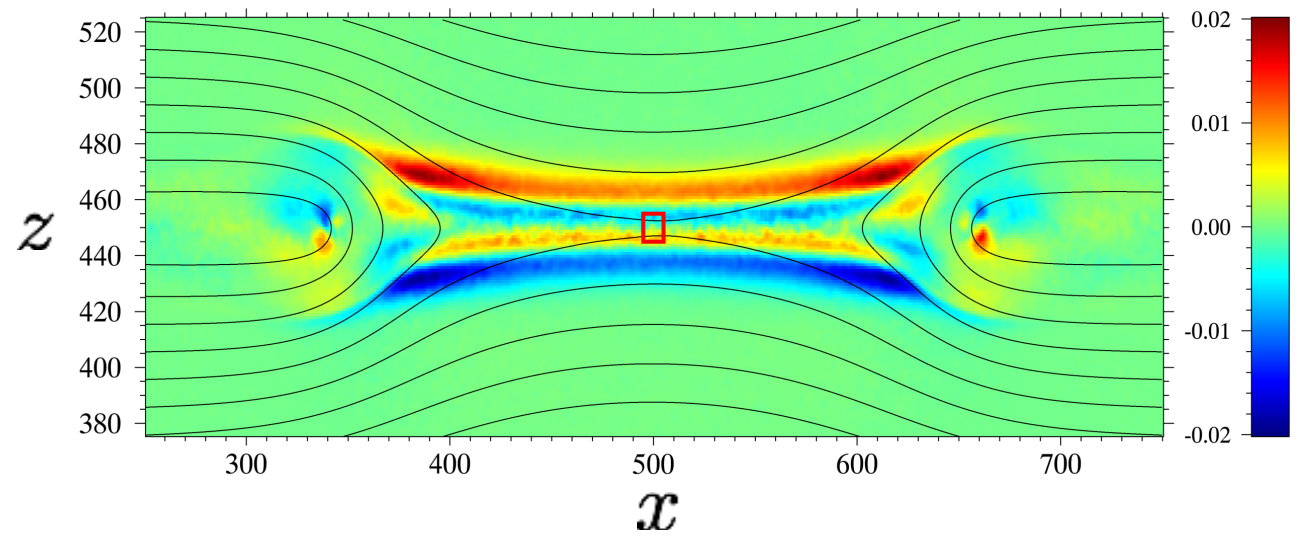

Figure 4. Spatial profiles of (a) the pressure tensor term $P_{\text {eyz }}$ and (b) the heat-flow term $Q_{e y z}$ at $t=85$.

Shown in Figure 4 are spatial profiles of $P_{e y z}$ and $Q_{e y z}$. The local momentum 
transport $P_{\text {eyz }}$ has a bipolar structure across the midplane (Fig. 4a). In the reconnection layer, the electrons travel in $-y$. Thus, the bipolar picture corresponds to the diffusion of the electron current-carrying momentum in the $\pm z$ directions, away from the midplane [36, 37]. This leads to the effective inertial effect via the $-\partial_{z} P_{\text {eyz }}$ term. The heat-flow term $Q_{e y z}$ gives a more complicated picture (Fig. 4 b). It exhibits a 4-layer structure of the outer bipolar layers and the inner bipolar layers. Around the midplane, the inner layers have the same signs as the bipolar $P_{\text {eyz }}$ layers. The outer layers have the opposite signs from the inner layers.

(a) 4-velocity distribution

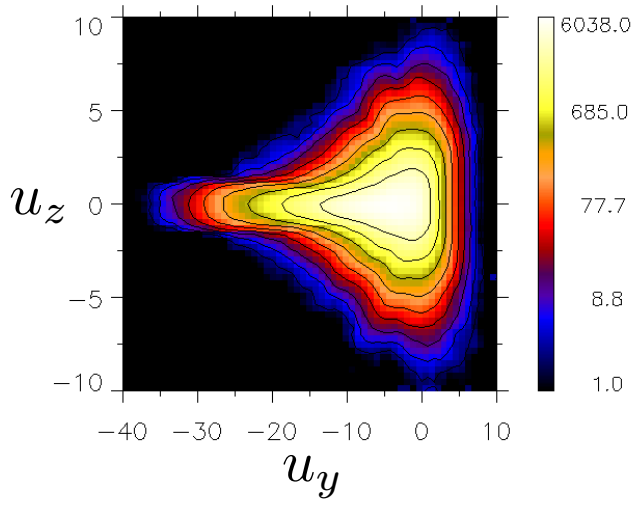

(b) Phase space diagram

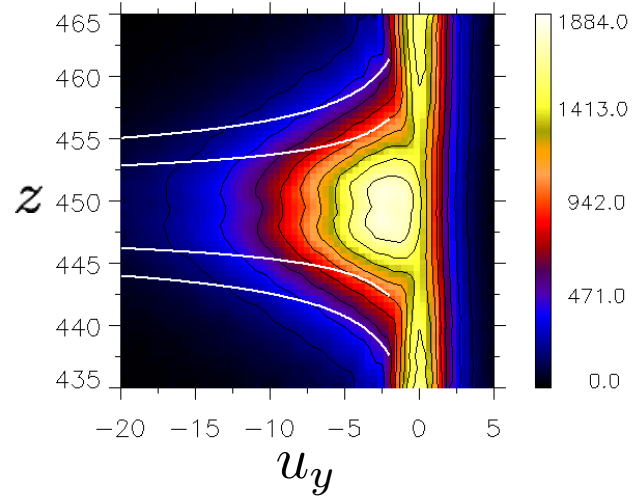

Figure 5. (a) Electron four-velocity distribution function $\left(u_{y}-u_{z}\right)$ and (b) electron phase-space diagram $\left(u_{y}-z\right)$ at $t=85$. The white lines indicate $\left|z-z_{0}\right| \propto \pm\left(-u_{y}\right)^{-1 / 3}$.

To understand the problem from microscopic viewpoints, we present an electron velocity distribution function (VDF) in $u_{y}-u_{z}$ around the $\mathrm{X}$ line in Figure 5 (a). The electrons are sampled in the red box in Figure $4(\mathrm{~b})((x, z) \in[495,505] \times[445,455])$ at $t=85$. The VDF is integrated in $u_{x}$. Although the VDF has a core component in the mildly relativistic domain $\left(-10 \lesssim u_{y} \lesssim 5\right)$, there exist highly-energetic electrons in the $-u_{y}$ direction, down to $u_{y} \approx-35$. This is due to the direct $y$-acceleration by the reconnection electric field $E_{y}$ 9. Figure 5(b) presents the electron phase-space diagram in $u_{y}-z$, integrated over $495 \leq x \leq 505$. The electrons are accelerated in $-y$ near the midplane $z \approx 450$. They travel in $-y$ through the Speiser motion [38, bouncing in $z$. Interestingly, the energetic electrons tend to be concentrated around the midplane. This is consistent with the relativistic feature of the Speiser motion. The Speiser $z$-bounce motion is a damped oscillation in $z$. Speiser 38 showed that the bounce width in $z$ decays like $z_{\max } \sim t^{-1 / 4}$ and that the kinetic energy is proportional to $\varepsilon \sim t^{2}$ in a simple 1D configuration. Thus we expect $z_{\max } \sim \varepsilon^{-1 / 8}$. In contrast, in the relativistic regime, it was recently shown that the bounce width shrinks much faster than in the nonrelativistic regime, $z_{\max } \sim \gamma^{-1 / 3} \sim \varepsilon^{-1 / 3}$ [39]. Since the highenergy electrons are directed to $-y$ (Fig. 5 (a)), we approximate $\left|u_{y}\right| \approx \varepsilon$, and then we expect $z_{\max } \propto \varepsilon^{-1 / 3} \approx\left(-u_{y}\right)^{-1 / 3}$, as indicated by the thin white lines in Figure $5(\mathrm{~b})$. The phase-space distribution is in agreement with the relativistic shrinkage. The highenergy electrons are slightly more confined in $z$ than predicted, but this is probably because the reconnected magnetic field $B_{z}$ ejects particles in the $\pm x$ directions, and because the system is still developing. 
The inner bipolar layer of $Q_{e y z}$ (Fig. 4 4 ) corresponds to the typical bounce width in $z$ (Fig. 5b). Inside this meandering channel, there exist a huge amount of the high-energy electrons, as evident in the VDF (Fig. 5a). These energetic electrons lead to the heat flow in the $-y$ direction. They follow the meandering motion and then scatter the $-y$-momentum inside the meandering channel: upward in the upper half $\left(Q_{e y z}<0\right)$ and downward in the lower half $\left(Q_{e y z}>0\right)$. Meanwhile, in the outer $Q_{e y z}$ layer, the electrons travel from the upstream regions toward the midplane, and then start to be accelerated in $-y$ as they approach the midplane. In addition, some meandering electrons travel through the outer $Q_{e y z}$ layer, but their orbits shrink to the midplane as they are accelerated in $-y$. As a result, the $-y$-momentum of the energetic electrons are transported toward the meandering channel: downward in the upper half $\left(Q_{e y z}>0\right)$ and upward in the lower half $\left(Q_{e y z}<0\right)$ (Fig. 4(b)).

The spacial gradient of the heat-flow tensor results in the heat-flow inertial effect in the Ohm's law (Eq. 8p. Around the midplane, between the inner $Q_{\text {eyz }}$ layers, the outward transport of the current-carrying heat-flow leads to the inertial effects in the form of $-\partial_{z} Q_{e y z}>0$ (Fig. 3). The underlying physics is essentially the same as the momentum diffusion in the $-\partial_{z} P_{\text {eyz }}>0$ term [4]. Outside there, between the inner and outer $Q_{e y z}$ layers, the heat flows in the $-y$ direction are transported from the midplane and from the inflow regions. This results in the convergence of the currentcarrying heat-flow, and therefore the heat-flow inertial term has the opposite sign, $-\partial_{z} Q_{e y z}<0$. Farther outside, between the outer $Q_{e y z}$ layers and the inflow regions $(430 \lesssim z \lesssim 435$ and $465 \lesssim z \lesssim 470$ ), the divergence of the current-carrying heat-flow again leads to an effective inertial effect in the form of $-\partial_{z} Q_{e y z}>0$. The heat-flow inertial term changes the sign 5 times, because of the 4-layer structure of $Q_{e y z}$.

In this case, the heat-flow inertial term $\left(\nabla \cdot Q^{i j}\right)$ plays a smaller role than the divergence of the pressure tensor term $\left(\nabla \cdot P^{i j}\right)$ to sustain the reconnection electric field $E_{y}$ at the $\mathrm{X}$ line. This is reasonable, because the number of energetic electrons are much smaller than that of the core electrons (Figs. 5(a,b)). However, importantly, the heat-flow inertial term is nonzero and it plays a similar role as the pressuretensor term. It remains nonzero at the midplane across the entire reconnection site $(370 \lesssim x \lesssim 630)$, as evident in the relevant $Q_{\text {eyz }}$ layers (Fig. 5 ).

\section{Energy balance}

Using the Eckart decomposition, we further study the energy balance during the reconnection process. The energy balance is described by the $\alpha=0$ part of the following equation,

$$
\partial_{\beta}\left(T_{(p)}^{\alpha \beta}+T_{(e)}^{\alpha \beta}+T_{(E M)}^{\alpha \beta}\right)=0,
$$

where $T_{(E M)}^{\alpha \beta}$ is the stress-energy tensor by the electromagnetic field. We evaluate the energy flux part (the $0 i$ components) of the plasma stress-energy tensor,

$$
\begin{aligned}
T^{0 i} & =\mathcal{E} u^{0} u^{i}+P^{0 i}+Q^{0 i} \\
& =N^{i}+(\gamma-1) N^{i}+\left[(\mathcal{E}-n) u^{0} u^{i}+P^{0 i}\right]+Q^{0 i} .
\end{aligned}
$$

In Equation 15, we rewrite the right hand side by using the matter flow $N^{i}=n u^{i}$. The second term in the right hand side corresponds to the bulk kinetic energy flux in the nonrelativistic case. The third term between the square brackets is a combination of the flow of the plasma internal energy and the work associated with the pressure. 
Thus this corresponds to the enthalpy flux. The last heat-flow term carries an additional energy flow. The $0 i$ part of the electromagnetic stress-energy tensor is the Poynting flux. In our PIC simulation, we consider a square box at the center $((x, z) \in[425,575] \times[410,490])$, as indicated in Figure 2(a). Then we integrate the inflow energy flux $\left( \pm\left[T_{(p)}^{0 z}+T_{(e)}^{0 z}+T_{(E M)}^{0 z}\right]\right)$ along the upper and bottom sides and the outflow energy flux $\left( \pm\left[T_{(p)}^{0 x}+T_{(e)}^{0 x}+T_{(E M)}^{0 x}\right]\right)$ along the left and right sides.

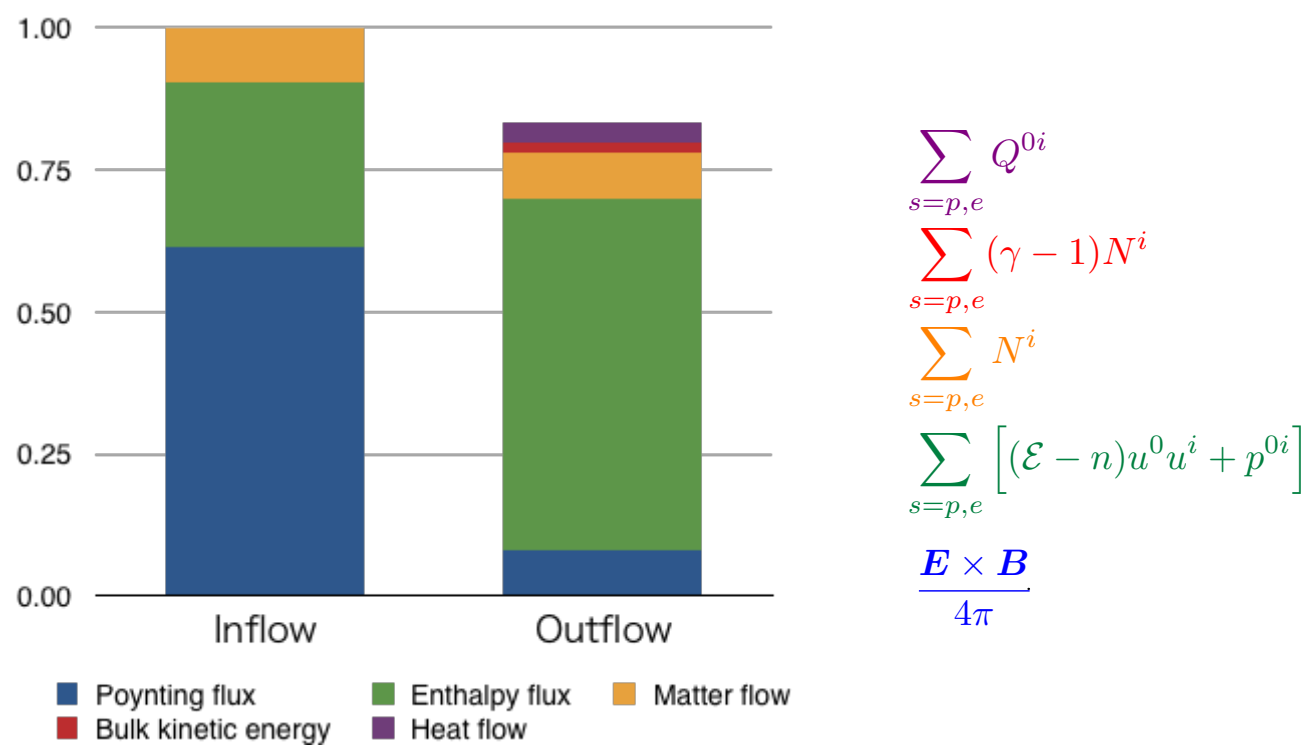

Figure 6. The composition of the incoming and outgoing energy fluxes around the reconnection site.

Figure 6 shows the composition of the energy fluxes at $t=85$. The positron and electron fluxes are combined, because they are essentially equal. The fluxes are normalized by the total inflow energy flux, which is larger than the total outflow flux at this point. The Poynting flux is the main energy carrier in the inflow region. The enthalpy flux is the second carrier. This is larger than the matter flow, because the plasma temperature is initially relativistic $T=1$. The classical bulk kinetic energy and the heat flow are negligible.

The composition of the outflow energy flux is very different from that of the inflow flux. The enthalpy flux is the biggest energy carrier. This tells us that the reconnection process convert the electromagnetic energy in the upstream region into the plasma heat and that the relativistically hot plasma carries the energy in the form of the enthalpy flux in the downstream region. The matter flow is comparable with that in the inflow region. The bulk kinetic energy flux carries some amount of the energy. In this case, as stated before, plasmas inside the reconnection layer become relativistically hot $T \gtrsim 1.5$, and therefore the enthalpy flux exceeds the combination of the matter flow and the bulk kinetic energy flux, i.e., $4 n T u^{0} u^{i} \gg n u^{0} u^{i}$. The heat flow carries more energy than the bulk kinetic energy flux. The Poynting flux is largely carried by the oblique magnetic field lines away from the midplane. 


\section{Discussion and Summary}

We have revisited the composition of the reconnection electric field in relativistic pair plasma reconnection. Using the Eckart decomposition 29, we have formulated the relativistic kinetic Ohm's law. Analyzing the PIC simulation data, we have basically confirmed the conventional picture that the divergence of the pressure tensor balances the reconnection electric field, in agreement with nonrelativistic studies 2, 3, 4, and the previous study with different formulation [13. We have further found that the relativistic temperature enhances the bulk inertial effect and that the new heat-flow inertial effect arises from the Speiser motion of the energetic electrons. Note that the heat flow is different from the heat conduction, because the inertial effect arises from the off-diagonal components of $Q$, while the heat conduction is usually driven by the temperature gradient, i.e., $\nabla T$. The heat conduction is unable to sustain the reconnection electric field $E_{y}$ in the $2 \mathrm{D}$ system of $\partial_{y}=0$. Additional analysis on the VDF, the phase-space diagram, and the spatial distribution of $Q_{e y z}$ suggests that the heat-flow inertial effect stems from the Speiser particle acceleration around midplane, which is a robust feature of relativistic reconnection. In this sense, the underlying physics of the new term is similar to that of the conventional pressure-tensor term, which stems from the Speiser motion of the bulk populations. The 4-layer structure of $Q_{e y z}$ leads to the characteristic profile of the heat-flow inertial term.

We have also studied the energy balance during the reconnection process. We have found that the incoming Poynting flux is transferred to the enthalpy flux in the outflow region. The heat flow carries more energy than the matter flux in the outflow region, however, the enthalpy flux is a major carrier of the outgoing energy. This is consistent with the previous two-fluid simulation [28] and extends previous nonrelativistic results $[24,25,26,27$. It is reasonable that the upstream magnetic energy is transferred to the plasma internal energy, a energy of a high-entropy state, through a dissipative process like magnetic reconnection.

There are some limitations in our analysis. First, our system is still evolving in time. The temporal part is non-negligible in the Ohm's law (Fig. 3), and the inflow and outflow energy fluxes are not equal in the energy balance (Fig. 6p. Second, our box size may not be ideal to discuss the energy balance, because it is shorter in $x$ than the extent of the dissipation region (Figs. 2a,b). These two issues may be overcome by using a larger simulation domain. In a larger domain, the system might reach a quasisteady stage, so that we can rule out time-dependent effects. The outflow speed might reach the upstream Alfvén speed, as suggested by the theory 40, and MHD simulations [41]. Then, the bulk kinetic energy flux will carry a larger amount of the energy in the outflow region. However, even in this case, the reconnection site started to generate secondary islands after $t \gtrsim 85$. In our experience, the number of particles in a cell often controls the onset of the island formation, but we used a large number of particles in this study. Considering that larger PIC simulations often exhibit secondary islands, it may not be necessary to stick to the quasi-steady picture. Third, we have analyzed only one run. In astrophysical applications, we expect that the reconnection occurs in extreme situations, in which the initial magnetic energy vastly exceeds the plasma energy. The upstream leptons are often supposed to be cold, due to the synchrotron cooling. In such extreme cases, since more energy will be transferred to energetic particles, and since their energy easily exceeds the typical thermal energy, the heat-flow terms could be more pronounced, both in the Ohm's law and in the energy balance. For example, if the heat-flow inertial term replaces the 
pressure-tensor term in the Ohm's law, a widespread belief that the pressure-tensor term is essential at the X line [1, 4, will be challenged. These issues are left for future investigations.

We have employed the Eckart velocity as the fluid velocity in this work. If we employed Landau velocity $\left(u_{(L)}^{\alpha}\right)$ instead, since $q^{\alpha}=0$ in Equation 5 , the heat-flow inertial term in the Ohm's law and the heat-flow term in the energy flux disappear. However, the Eckart velocity $\left(u_{(E)}^{\alpha}\right)$ should be used in the right hand side of the energymomentum equation (Eq. 7), because the plasma number flow carries the electric current. To evaluate the Ohm's law (Fig. 3), we have to use both $u_{(E)}^{\alpha}$ and $u_{(L)}^{\alpha}$, or to introduce an additional flow, $u_{(C)}^{\alpha} \equiv u_{(E)}^{\alpha}-u_{(L)}^{\alpha}$. In the energy balance, it is less meaningful to discuss the matter flow $N^{i}=n u_{(E)}^{i}$ and relevant quantities by using the Landau-frame quantities. The energy dissipation (Eq. 12) also requires the number density's flow [35, i.e., the Eckart velocity. Based on these considerations, we prefer the Eckart-frame approach to evaluate the PIC results.

In summary, using the Eckart decomposition, we have analyzed the kinetic Ohm's law and the energy balance in relativistic reconnection in a pair plasma. We have basically confirmed previous expectations that the pressure-tensor term is responsible for the reconnection electric field $E_{y}[13]$ and that the relativistic enthalpy-flux carries the outgoing energy, but additional roles by the relativistic heat-flow terms are discovered. In addition to these results, it is meaningful to demonstrate the diagnostics for the relativistic PIC data. Then it will be possible to cross-compare relativistic PIC simulations, relativistic fluid simulations, and relativistic MHD theories.

\section{Acknowledgments}

This work was supported by Grant-in-Aid for Young Scientists (B) 25871054 and Grant-in-Aid for Scientific Research (C) 17K05673 from the Japan Society for the Promotion of Science (JSPS).

\section{Appendix A. Eigenvalue properties for the Landau frame}

Since the energy flow disappears in the Landau frame, $q^{\mu}\left(u_{(L)}^{\mu}\right)=0$, the Landau-frame four-velocity $u_{(L)}^{\mu}$ satisfies

$$
T_{\delta}^{\alpha} u_{(L)}^{\delta}=\left(-u_{(L) \beta} T^{\beta \gamma} u_{(L) \gamma}\right) u_{(L)}^{\alpha} .
$$

This indicates that $u_{(L)}^{\mu}$ is an eigenvector of a $4 \times 4$ matrix $\left(T_{\delta}^{\alpha}\right)$. The factor on the right hand side is equivalent to the energy density in the Landau frame, and therefore the eigenvalue is negative, $-\mathcal{E}\left(u_{(L)}^{\alpha}\right)<0$.

Let us further consider a space-like vector $x^{\prime \alpha}=\left(0, x^{\prime i}\right)$ in the Landau frame. Since $T^{i 0}=T^{\prime 0 i}=0$, there exists three vectors that satisfy the following relation for a scalar $p^{\prime}$,

$$
T^{\prime \alpha \beta} x_{\beta}^{\prime}=p^{\prime \alpha \beta} x_{\beta}^{\prime}=p^{\prime} x^{\prime \alpha},
$$

where $p^{\prime}=p_{1,2,3}^{\prime}$ and $x^{\prime}=x_{1,2,3}^{\prime}$ are the eigenvalues and eigenvectors of the pressure tensor. Transforming Equation A.2 to the observer frame,

$$
\Lambda_{\mu}^{\alpha} T^{\prime \mu \beta} g_{\beta \nu} x^{\prime \nu}=p^{\prime}\left(\Lambda_{\mu}^{\alpha} x^{\prime \mu}\right)=p^{\prime} x^{\alpha}
$$

Here we used $g_{\beta \nu} x^{\prime \nu}=x_{\beta}^{\prime}$, because $x^{\prime}$ has no time component. We also use the definition of the Lorentz transform, $\Lambda_{\beta}^{\gamma} g_{\gamma \delta} \Lambda_{\nu}^{\delta}=g_{\beta \nu}$,

$$
\Lambda_{\mu}^{\alpha} T^{\prime \mu \beta} \Lambda_{\beta}^{\gamma} g_{\gamma \delta} \Lambda_{\nu}^{\delta} x^{\prime \nu}=T_{\gamma}^{\alpha} x^{\gamma}=p^{\prime} x^{\alpha} .
$$


This tells us that Equation A.1 has three positive eigenvalues and the space-like eigenvectors, corresponding to the eigenvalues of the pressure tensor and the spatial axises in the Landau frame. From their definitions in the Landau frame,

$$
\mathcal{E}^{\prime} \equiv \int f^{\prime}\left(u^{\prime}\right) \gamma^{\prime} \gamma^{\prime} \frac{d^{3} u^{\prime}}{\gamma^{\prime}}>\int f^{\prime}\left(u^{\prime}\right) u^{\prime 2} \frac{d^{3} u^{\prime}}{\gamma^{\prime}}=p_{1}^{\prime}+p_{2}^{\prime}+p_{3}^{\prime}
$$

it is obvious that the negative eigenvalue $-\mathcal{E}^{\prime}$ has the biggest absolute value among the four eigenvalues.

In addition, the energy density in the observer frame yields

$$
T^{00}=\Lambda_{\alpha}^{0} \Lambda_{\beta}^{0} T^{\prime \alpha \beta}=\gamma^{2} \mathcal{E}^{\prime}+\left(P^{\prime} \vec{u}_{(L)}\right) \vec{u}_{(L)}
$$

where $\vec{u}_{(L)}=\left(u^{1}, u^{2}, u^{3}\right)$ is the spatial part of the Landau four-velocity. By rewriting $\vec{u}(L)=a_{1}{\overrightarrow{x^{\prime}}}_{1}+a_{2}{\overrightarrow{x^{\prime}}}_{2}+a_{3}{\overrightarrow{x^{\prime}}}_{3}$ with the unit eigenvectors ${\overrightarrow{x^{\prime}}}_{1,2,3}$, we find

$$
T^{00}=\left(1+a_{1}^{2}+a_{2}^{2}+a_{3}^{2}\right) \mathcal{E}^{\prime}+\left(p_{1}^{\prime} a_{1}^{2}+p_{2}^{\prime} a_{2}^{2}+p_{3}^{\prime} a_{3}^{2}\right) \geq \mathcal{E}^{\prime} .
$$

This tells us that the energy density always increases, by the Lorentz transformation from the Landau frame. In other words, the Landau frame is a frame that minimizes the energy density.

\section{References}

[1] Vasyliunas, V. M. 1975, Reviews of Geophysics and Space Physics, 13, 303

[2] Cai, H. J. and Lee, L. C. 1997, Phys. Plasmas, 4, 509

[3] Hesse, M., Schindler, K., Birn, J., \& Kuznetsova, M. 1999, Phys. Plasmas, 6, 1781

[4] Hesse, M., Neukirch, T., Schindler, K., Kuznetsova, M., \& Zenitani, S. 2011, Space. Sci. Rev. , 160,3

[5] Uzdensky, D. A. 2011, Space. Sci. Rev. , 160, 45.

[6] Blackman, E. G. \& Field, G. B. 1993, Phys. Rev. Lett., 71, 3481

[7] Gedalin, M. 1996, Phys. Rev. Lett., 76, 3340

[8] Meier, D. L. 2004, Astrophys. J., 605, 340

[9] Zenitani, S. \& Hoshino, M. 2001, Astrophys. J., 562, L63

[10] Jaroschek, C. H., Treumann, R. A., Lesch, H., \& Scholer, M. 2004, Phys. Plasmas, 11, 1151

[11] Zenitani, S. \& Hoshino, M. 2007, Astrophys. J., 670, 702

[12] Zenitani, S. \& Hoshino, M. 2008, Astrophys. J., 677, 530

[13] Hesse, M. \& Zenitani, S. 2007, Phys. Plasmas, 14, 112102

[14] Liu, W., Li, H., Yin, L., Albright, B. J., Bowers, K. J., \& Liang, E. P. 2011, Phys. Plasmas, 18, 052105

[15] Bessho, N. \& Bhattacharjee, A. 2012, Astrophys. J., 750, 129

[16] Cerutti, B., Werner, G. R., Uzdensky, D. A., \& Begelman, M. C. 2013, Astrophys. J., 770, 147

[17] Sironi, L. \& Spitkovsky, A. 2014, Astrophys. J., 783, L21

[18] Melzani, M., Walder, R., Folini, D., Winisdoerffer, C., \& Favre, J. M. 2014, A\&A , 570, 111

[19] Liu, Y.-H., Guo, F., Daughton, W., Li, H., \& Hesse, M. 2015, Phys. Rev. Lett., 114, 095002

[20] Werner, G. R., Uzdensky, D. A., Begelman, M. C., Cerutti, B., \& Nalewajko, K. 2016, submitted to Mon. Not. R. Astron. Soc., arXiv:1612.04493

[21] Wright, T. P. \& Hadley, G. R. 1975, Phys. Rev. A, 12, 686

[22] Birn, J. \& Hesse, M. 2005, Ann. Geophys., 23, 3365.

[23] Birn, J. \& Hesse, M. 2009, Ann. Geophys., 27, 1067.

[24] Aunai, N., Belmont, G., \& Smets, R. 2011, Phys. Plasmas, 18, 122901.

[25] Yamada, M., Yoo, J., Jara-Almonte, J., Daughton, W., Ji, H., Kulsrud, R. M., Myers C. E. 2015, Phys. Plasmas, 22, 056501

[26] Yamada, M., Yoo, J., and Zenitani, S., 2016, Astrophysics and Space Science Library, 427, 143.

[27] Lapenta, G., Goldman, M. V., Newman, D. L., \& Markidis, S. 2017, Plasma Phys. Control. Fusion, 59, 014019

[28] Zenitani, S., Hesse, M., \& Klimas, A. 2009, Astrophys. J., 705, 907

[29] Eckart, C 1940, Physical Review, 58, 919

[30] Landau, L. D. \& Lifshitz, E. M. 1959, "Fluid Mechanics", Oxford, 2nd edition (1987), §127

[31] Synge, J. L. 1956, "Relativity: the special theory", North-Holland, Chapter 8 
[32] Harris, E. G., 1962, Nuovo Cimento, 23, 115

[33] Hoh, F. C. 1966, Phys. Fluids, 9, 277

[34] Zenitani, S. 2015, Phys. Plasmas, 22, 042116

[35] Zenitani, S., Hesse, M., Klimas, A., \& Kuznetsova, M. 2011, Phys. Rev. Lett., 106, 195003

[36] Cai, H. J. Ding, D. Q., \& Lee, L. C. 1994, J. Geophys. Res., 99, 35.

[37] Fujimoto, K. \& Sydora, R. D. 2009, Phys. Plasmas, 16, 112309

[38] Speiser, T. W. 1965, J. Geophys. Res., 70, 4219, doi:10.1029/JZ070i017p04219.

[39] Uzdensky, D. A., Cerutti, B., \& Begelman, M. C. 2011, Astrophys. J., 737, L40

[40] Lyubarsky, Y. 2005, Mon. Not. R. Astron. Soc., 358, 113

[41] Zenitani, S., Hesse, M., \& Klimas, A. 2010, Astrophys. J., 716, L214 